\title{
A Configurable Tool for Generating and Managing Reusable Simulation Model
}

\author{
Hai-bo $\mathrm{MA}^{*}$ and Yi-ping YAO \\ College of Information System and Management, National University of Defense \\ Technology, Changsha, Hunan, China \\ *E-mail: mahaibo168@126.com
}

Keywords: Model reuse, Parameter-configurable structure, Reusable simulation model.

\begin{abstract}
How to generate a new suitable reusable model from previous reusable model is a key issue in model reuse. Traditional model reuse focus much on how a specific function model to be reused in new applications, however, lacks consideration about reusability efficiency of reusable simulation models of the same class. Some models compose of similar computational functions with different combination, they have to be programmed and VV\&A repeatedly, which cause unnecessary development cost. To solve this problem, this paper proposed a parameter-configurable structure for the same class reusable simulation model. Based on the structure, this paper designed and implemented a configurable tool for generating reusable simulation models of the same class, and diversiform simulation model products can be configured to generate satisfying different requirement. The tool gives a pragmatic technical means to develop reusable models, which proved to be an efficient solution to avoid repeat modeling and help reduce cost when developing new simulation applications.
\end{abstract}

\section{Introduction}

With the increasing scale of simulation, the development costs increase dramatically. One of the challenges is model reuse and model management while reducing the development costs. Reuse is "using a previously developed asset again, either for the purpose for which it was originally developed or for a new purpose or in a new context" [1]. Reusable component model can be selected from a model resource library to assemble new applications, thus reducing both time and costs compared with a new development for models [2]. Despite substantial efforts, almost always well-intentioned and well-executed, reuse and composability have not been fully achieved; indeed, they were recently described as "still biggest simulation challenge" [3] that in return influenced efficiency of reuse. Traditional model reuse focus much on how a specific function model reusing in new applications, however, lacks consideration about reusability efficiency of reusable simulation models of the same class. As a result, some models have to be programmed and VV\&A repeatedly, which cause unnecessary development cost. There are also unavoidable theoretical obstacles; it has been formally proven that the composition of two valid models cannot be assumed to be valid [4].

In contemporary practice, there are daunting engineering requirements in military simulation applications, i.e., a naval vessel component, was developed and VV\&A to integrate an simulation application. Then in the other projects, there may needs other type of naval vessels, such as X-type cruiser, Y-type destroyer. In these occasion, we desire to build the new naval vessel model from the previous similar model. However, these models usually contain different computational modules, and each module may 
have various performance. If rebuild the required models based on the previous one, the simulation model itself is still largely hand-built and ad hoc, with all the negatives implied, including excessive time and cost for development, inconsistency in application and difficulty of reuse. Therefore, how to generate a new suitable reusable model from previous reusable model is an import challenge to be resolved.

This paper proposed a parameter-configurable structure for reusable simulation model of the same class. Based on the structure, the paper designed and implemented a configurable tool for generating and managing reusable simulation models of the same class, in which a prototype reusable model with full function-set is developed once time, and diversiform simulation models could be configured to generate with different functions combination.

\section{Related Work}

In the current research, there are basically two ways to realize model reuse, they are combination based modeling thought and base class based modeling thought.

Combination based modeling draw lessons from DEVS[5] idea. Some models are built with a certain constraint specification, which is called "atomic model", such as radar model and missile model. These atomic models are used to assemble a destroyer ship simulation model according to performance requirement. Among them, the communication port is required for interaction between different sub models, and interaction logic is organized in visualization tools. However, this approach is potentially inefficient and difficult to implement. On the one hand, if a complex ship simulation model needs different type radar model, missile model and damage evaluation model, it will leads to explosion problem of resource combination. On the other hand, it is difficult to define the granularity of reusable simulation model.

Compared to the combination based modeling, base class based modeling thought draw on experience of inheritance thought in object oriented programming. New model is developed based on the basis of the prototype model. The model is reuse the previous code framework with only small amount of code modification. The base class based modeling is mainly embodied in some specific simulation modeling and development platforms. Typical products such as FLAMES (Analysis Modeling, and Exercise System Flexible) [6]. They build a base class for each type of model, and users can inherit the base classes to develop their own models. However, the model base class is based on its specific simulation platform interface design that needs manual development rules, therefore they do not have good reusability.

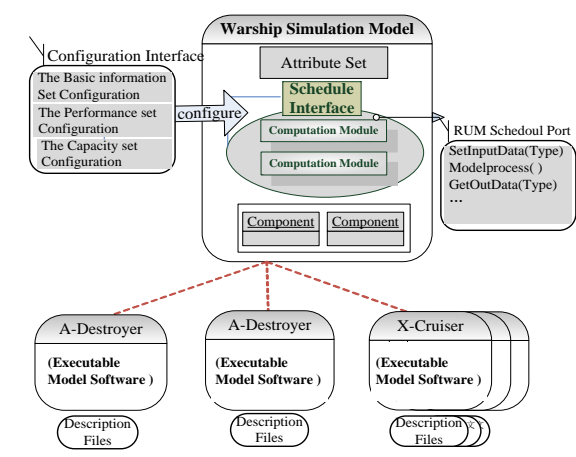

Fig. 1. The parameterization-configurable Structure. 


\section{Parameter-configurable Structure for Reusable Simulation Model of the Same Class}

To provide an efficient way for generating a new suitable reusable model from previous reusable model, this paper proposed parameter-configurable structure for reusable simulation model of the same class. Modeling based on prototype supporting parametric configuration is the combination of the above two ways for model reuse, which achieves once development and multiple reuse [7].

The framework composes of three parts: a visual configuration interface; model prototype framework; executable model software product. Fig.1 shows the parameterconfigurable structure for reusable simulation model of normal naval vessels. All the performance data is designed to support configuration in visual interface. The configured data is recorded in the configuration file and parsed in the prototype framework. The warship prototype model software can be easily and quickly configured to generate many specific similar warship models with specific performance such as A-Destroyer, X-Destroyer as well as Y-Cruiser. Compared to traditional model reuse, the parameter-configurable framework provide a much more efficient means achieving model reuse.



Fig. 2. The parameterization-configurable Model Prototype

The model prototype framework is developed as basic code class that inside implements essential functions of naval vessels, which mainly contains cruse move, radar detection, senor detection, ECM, missile attack, artillery attack, torpedo attack and so on. All these function modules are developed as independently atomic models 
in DEVS that encapsulate domain computation into input-output functions that is called computation module. The Fig. 2 gives the introduction of implementation for the prototype framework of naval vessels[7].. Moreover, to achieve reusability, the model needs to be platform independent. The passive-schedule common-service interface is designed to avoid reusable model involves in the specific platforms or applications. Computation modules is organized by logicControl module through common-service interfaces. The passive-schedule interface decides that the model only focus on its own business and provide computation service to schedulers, so that the model is completely independent.

\section{Tool for Developing Reusable Simulation Model}

Based on parameter-configurable structure, this paper implemented a configurable tool for generating reusable naval vessels simulation models.

User configures different performance parameters for the specific warship simulation model through the tool interface, and then it will generate executable model software with model product. Compared to traditional ways to develop these models by manual coding, the parameterization-configurable tool provides high efficiency for the warship model reuse, which dramatically decrease time of developing simulation applications while supporting models once developed but multiple reused adapting to varied function requirement. Fig.3[7]indicate that parameterization-configurable tool can well satisfy requirement for reusable simulation model.

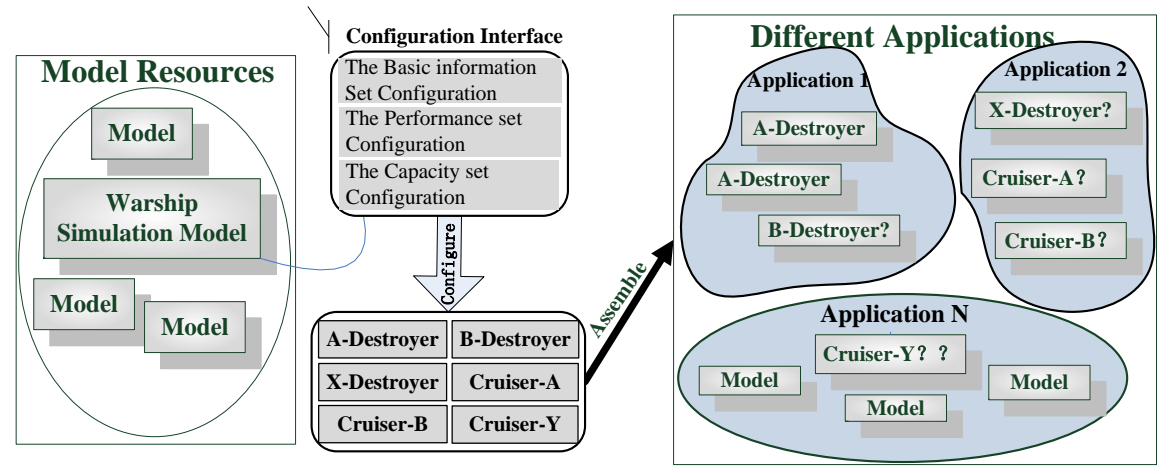

Fig.3. The Parameter-configurable Reusable Model for developing Simulation Applications

\section{Conclusions}

How to generate a new suitable reusable model from previous reusable model is a key issue in model reuse. Traditional ways exists repeated modeling for similar models that cause unnecessary development cost. This paper proposed a navel parameterconfigurable structure and designed a configurable tool for generating and managing reusable simulation models, by which diversiform simulation models can be configured to generate satisfying different requirement. The tool gives a pragmatic technology mains for developing reusable simulation models in some specific domain, which proved to be an efficient solution to avoid repeat modeling and help reduce development cost. 


\section{Acknowledgments}

This work was supported by National Natural Science Foundation of China (No.61170048) and Research Project of State key Laboratory of High Performance Computing of National University of Defense Technology (No.201303-05).

\section{References}

[1] M. D. Petty, K. L. Morse, W. C. Riggs, P. Gustavson, and H. Rutherford, "A Reuse Lexicon: Terms, Units, and Modes in M\&S Asset Reuse", Proceedings of the Fall 2010 Simulation Interoperability Workshop, Orlando FL,September 20-24 2010.

[2] Workshop Participants,Workshop on Research Challenges in Modeling \& Simulation for Engineering Complex Systems M\&S Challenges, January 13-14, 2016.

[3] S. J. E. Taylor, A. Khan, K. L. Morse, A. Tolk, L. Yilmaz, J. Zander, and P. J. Mosterman, "Grand challenges for modeling and simulation: simulation everywherefrom cyberinfrastructure to clouds to citizens",SIMULATION: Transactions of the Society for Modeling and Simulation International, Vol. 91, No. 7, July 2015,pp. 648665.

[4] E. W. Weisel, R. R. Mielke, and M. D. Petty, "Validity of Models and Classes of Models in Semantic Composability", Proceedings of the Fall 2003 Simulation Interoperability Workshop, Orlando FL, September 14-19, 2003, pp. 526-536.

[5] JAMMALAMADAKA R, ZEIGLER B P, ZHANG M, et al. Complex system simulation: DEVS implementation of the valley fever model[C]//Proceedings of the 2007 spring simulation multiconference, Norfolk, Virginia, 2007:316-319.

[6] Ternion Corperation. FLAMES Makes Complex Systems Analysis Simple.

[7] Haibo Ma, Yiping Yao, "Warship Reusable Component Model Development Approach for Parallel and Distributed Simulation" in Proceedings of the 16th Asia Simulation Conference and the 2016 Autumn Simulation Multi-Conference (AsiaSim/SCS2016), October 2016. 